Article

\title{
The New New-Nacci Method for Calculating the Roots of a Univariate Polynomial and Solution of Quintic Equation in Radicals
}

\author{
Ilija Tanackov ${ }^{1, *}$, Ivan Pavkov ${ }^{2}$ and Željko Stević ${ }^{3}$ \\ 1 Faculty of Technical Sciences, University of Novi Sad, Trg Dositeja Obradovića 6, 21000 Novi Sad, Serbia \\ 2 Faculty of Mathematics and Computer Science, Alfa BK University, Palmira Toljatija 3, \\ 11000 Belgrade, Serbia; ivan.pavkov@alfa.edu.rs \\ 3 Faculty of Transport and Traffic Engineering Doboj, University of East Sarajevo, Vojvode Mišića 52, \\ 74000 Doboj, Bosnia and Herzegovina; zeljkostevic88@yahoo.com or zeljko.stevic@sf.ues.rs.ba \\ * Correspondence: ilijat@uns.ac.rs
}

Received: 15 March 2020; Accepted: 10 April 2020; Published: 8 May 2020

\begin{abstract}
An arbitrary univariate polynomial of $n$th degree has $n$ sequences. The sequences are systematized into classes. All the values of the first class sequence are obtained by Newton's polynomial of $n$th degree. Furthermore, the values of all sequences for each class are calculated by Newton's identities. In other words, the sequences are formed without calculation of polynomial roots. The New-nacci method is used for the calculation of the roots of an $n$ th-degree univariate polynomial using radicals and limits of successive members of sequences. In such an approach as is presented in this paper, limit play a catalytic-theoretical role. Moreover, only four basic algebraic operations are sufficient to calculate real roots. Radicals are necessary for calculating conjugated complex roots. The partial limitations of the New-nacci method may appear from the decadal polynomial. In the case that an arbitrary univariate polynomial of $n$th degree $(n \geq 10)$ has five or more conjugated complex roots, the roots of the polynomial cannot be calculated due to Abel's impossibility theorem. The second phase of the New-nacci method solves this problem as well. This paper is focused on solving the roots of the quintic equation. The method is verified by applying it to the quintic polynomial with all real roots and the Degen-Abel polynomial, dating from 1821.
\end{abstract}

Keywords: nonlinear approximate method; quintic equation; Pentanacci sequence; Abel's impossibility theorem

\section{Introduction}

Leonardo Pisano's (born c. 1170, died after 1240, posthumously known as Fibonacci) "Liber Abbaci", from 1202, is a Prometheus book of Mathematics. In the chapter "Quot paria coniculorum in uno anno ex uno paro germinentur", the famous sequence $0,1,1,2,3,5,8$ is presented. In 1597, Michael Maestlin (1550-1631), professor of the University of Tibingen, published the first known approximation of the De divina proportione. As a student of Michel Maestlin, Johannes Kepler (1571-1630) proved that the quotient of consecutive Fibonacci sequence numbers is constant. Therefore, the term "Kepler's limit" was used for the constant:

$$
\lim _{n \rightarrow+\infty} \frac{F(n+1)}{F(n)}=\phi=1.6180339 \ldots
$$

Kepler's contemporary Christopher Clavius (1538-1612), a German mathematician and astronomer, completed the Latin translation of "Elements" by Euclid of Alexandria. He recognized the identity 
of Euclid's "proportional division" and "Kepler's limit". In this way, a historical continuum, from Leonardo da Vinci (1452-1519) to the Parthenon and Phidias (500 BC-432 BC), was established.

At the same time, Gerolamo Cardano (1501-1576) and Lodovico de Ferrari (1522-1565), by their fundamental results, enabled further research in this field. With the achievements of François Viète (1540-1603), Isaac Newton (1642-1726), Jacques Philippe Marie Binet (1786-1856), and Eduard Lucas (1842-1891), the importance of Fibonacci's sequence was recognized. With Lucas' commitment [1], the sequence $0,1,1,2,3,5,8$ became known as the Fibonacci sequence. From Newton's second-degree polynomial with unit coefficients $x^{n}=x^{n-1}+x^{n-2}$, Binet's formula for the direct calculation of the Fibonacci sequence was derived.

John Pell (1611-1685) began exploring the sequence with coefficients $x^{n}=2 x^{n-1}+x^{n-2}$. However, a similar problem for the sequence defined by $x^{n}=x^{n-1}+2 x^{n-2}$ was solved by Ernst Jacobshtal (1882-1965) two centuries later. A general solution for Tribonacci and Quatronacci sequences of a univariate polynomial with arbitrary coefficients was developed by Leonard Carlitz (1907-1999). Carlitz's solution is applicable for the univariate polynomial up to the 5 th degree. The generalization of the sequences of higher degree was established only for sequences based on Newton's polynomial with unit coefficients $x^{n}=a_{1} x^{n-1}+a_{2} x^{n-2}+\ldots+a_{k} x^{n-k}, n \in \mathrm{N}, a_{1}=a_{2}=a_{k}=1$. One of the real roots always satisfies the condition:

$$
\phi_{1}+\frac{1}{\phi_{1}^{1}}=\phi_{2}+\frac{1}{\phi_{2}^{2}}=\phi_{3}+\frac{1}{\phi_{1}^{3}}=\ldots=\lim _{k \rightarrow \infty}\left(\phi_{k}+\frac{1}{\phi_{k}^{k}}\right)=2, \quad 1=\phi_{1}<\phi_{2}<\phi_{3}<\ldots<\phi_{\infty}=2
$$

In addition to the permanent "keeping the fire" in the Fibonacci Quarterly journal, the first decade of the 21st century had a significant impact on the research of the Fibonacci sequence, especially in the works of Alexey Stakhov [2-6], Sergio Falcon, and Angel Plaza [7-9]. Presently, the Fibonacci sequence is widely studied by numerous scientists worldwide, resulting in approximately 400 papers in indexed journals per year. However, research of higher sequences such as Tribonacci, Quatronacci, etc. are still rather rare [10-15]. The problem with Fibonacci sequence research is apparent in combinatorial identities: Cassini's identity, Catalan's identity, d'Ocagne's identity, Vajda's identity, Lucas' identity, or new identities [16,17]. All identities are proven for positive integers. Research of the Fibonacci sequences for negative integers is still open. The limit value from Fibonacci's sequence for negative integers-the second root of Newton's polynomial $x^{n}=x^{n-1}+x^{n-2}$ - has remained hidden for centuries:

$$
\lim _{n \rightarrow-\infty} \frac{F(n-1)}{F(n)}=-\frac{1}{\phi}=-0.6180339 \ldots
$$

The aforementioned identities precede investigations of the relationships between the roots of polynomials and recurrent "Fibonacci" sum [18-20] and other polynomial products in real [21,22] and complex domains [23].

In addition to the basic sequence based on the Newton's polynomial, there has been no exploration of sequences based on Vieta's rules. According to them, cubic equations have three sequences, quartic have four sequences, quintic have five sequences, etc. If the above holds, it can be proven that the limits inspired by the Kepler method, in fact, express values of polynomial roots, directly or indirectly. In addition to identity, there is also considerable research regarding the relationship between the roots of polynomials and Fibonacci sums and other polynomial products in real and complex domains.

Qubic and quartic univariate polynomials were solved in the 16th century. Isaac Newton and Joseph-Louis Lagrange (1736-1813) noticed that a quintic polynomial is insolvable in radicals [24]. Insolvability of general quintic equations in radicals was proved incompletely by Paolo Ruffini (1765-1822). Insolvability of general quintic polynomial and polynomials of a higher degree was completely proven by Niels Abel (1802-1829) in 1824. The result did not disable the existence of a special formula for each quintic polynomial. In 1826, he came to Paris, where he continued to work on his theorem on the insolvability of the quintic equation. He finished his theorem in October 1926 
and delivered it to French Academy of Sciences to be reviewed by Baron Augustin-Louis Cauchy (1789-1857). From Abel's biography, it can undoubtedly be concluded that the theorem and Abel's complete work was not adequately accepted in Paris (especially the paper revealing the double periodicity of elliptic functions). Furthermore, Abel caught tuberculosis in Paris. However, upon August Leopold Crelle's (1780-1855) invitation, Abel published his famous theorem (also known as Abel's impossibility theorem or Abel-Ruffini theorem) in 1926, in the first volume of Crelle's Journal. Without publishing it in Paris, Abel lost the financial support of the Norwegian government. His remaining years were marked by significant results which were published in Crelle's Journal (important work on elliptic modular functions). Abel died on 6 April 1829. Thanks to Adrien-Marie Legendre (1752-1833), Niels Abel was posthumously awarded the French academy Grand Prix for his work on elliptic functions, together with Carl Gustav Jacobi (1804-1851). However, even after this, Abel's theorem was not printed. After a formal request from the Norwegian government to the French academy in 1840, Abel's manuscript from 1826 was finally found among Cauchy's documents, and published. Nevertheless, the existence of general formulae for each quintic polynomial was indisposed by Evariste Galois (1811-1832).

The historical mathematical problem was alleviated by Charles Hermite with a solution of the general quintic equation in terms of elliptic modular functions in 1858. However, this approach did not enable further development of the sequences.

In the New-nacci method for calculating the roots of a univariate polynomial, all disadvantages of other methods are exceeded. Due to its historical significance, the new method is applied for the polynomial of the fifth degree, i.e., the quintic equation or Pentancci sequence.

The etymology of prefix "New" has dual meaning. It indicates that the method presented in the paper is completely original, as well as that our research is based on the work of Isaac Newton, on Newton identities.

Fibonacci, Lucas, Pell, Jacobi, and others generalized that Fibonacci sequences are, in fact, a Newton polynomial. Tribonacci, Quatronacci, Pentanacci, etc. sequences are Newton identities. Therefore, the denomination "nacci" of the method is suitable.

\section{Pentanacci Sequences}

\subsection{Preparation of Pentanacci Sequences}

All research of the Fibonacci sequence is based on the application of the Newton polynomial, which is defined by only one sequence. However, the number of sequences is much larger. The Fibonacci sequence has two classes, the Tribonacci sequence has three classes, etc. In general, the New-nacci sequence has $n$ classes. Let $p_{1}, p_{2}, p_{3}, p_{4}$ and $p_{5}$ be the roots of the quintic polynomial. Sequences are systematized using Vieta's formulae:

$$
x^{5}=\sum_{j=0}^{4} k_{j} x^{j}\left\{\begin{aligned}
+k_{4}= & p_{1}+p_{2}+p_{3}+p_{4}+p_{5} \\
-k_{3}= & p_{1} p_{2}+p_{1} p_{3}+p_{1} p_{4}+p_{1} p_{5}+p_{2} p_{3} \\
& +p_{2} p_{4}+p_{2} p_{5}+p_{3} p_{4}+p_{3} p_{5}+p_{4} p_{5} \\
+k_{2}= & p_{1} p_{2} p_{3}+p_{1} p_{2} p_{4}+p_{1} p_{2} p_{5}+p_{1} p_{3} p_{4}+p_{1} p_{3} p_{5} \\
& +p_{1} p_{4} p_{5}+p_{2} p_{3} p_{4}+p_{2} p_{3} p_{5}+p_{2} p_{4} p_{5}+p_{3} p_{4} p_{5} \\
-k_{1}= & p_{1} p_{2} p_{3} p_{4}+p_{1} p_{2} p_{3} p_{5}+p_{1} p_{2} p_{4} p_{5}+p_{1} p_{3} p_{4} p_{5}+p_{2} p_{3} p_{4} p_{5} \\
+k_{0}= & p_{1} p_{2} p_{3} p_{4} p_{5}
\end{aligned}\right.
$$


The relationship between the polynomial coefficients of $n$th degree and the roots of the quintic polynomial are named in accordance with combinations, sequences of I, II, III, IV, and V class (5):

$$
\begin{aligned}
P_{I}(n) & =p_{1}^{n}+p_{2}^{n}+p_{3}^{n}+p_{4}^{n}+p_{5}^{n} \\
P_{I I}(n) & =\left(p_{1} p_{2}\right)^{n}+\left(p_{1} p_{3}\right)^{n}+\left(p_{1} p_{4}\right)^{n}+\left(p_{1} p_{5}\right)^{n}+\left(p_{2} p_{3}\right)^{n} \\
& +\left(p_{2} p_{4}\right)^{n}+\left(p_{2} p_{5}\right)^{n}+\left(p_{3} p_{4}\right)^{n}+\left(p_{3} p_{5}\right)^{n}+\left(p_{4} p_{5}\right)^{n} \\
P_{I I I}(n) & =\left(p_{1} p_{2} p_{3}\right)^{n}+\left(p_{1} p_{2} p_{4}\right)^{n}+\left(p_{1} p_{2} p_{5}\right)^{n}+\left(p_{1} p_{3} p_{4}\right)^{n}+\left(p_{1} p_{3} p_{5}\right)^{n} \\
& +\left(p_{1} p_{4} p_{5}\right)^{n}+\left(p_{2} p_{3} p_{4}\right)^{n}+\left(p_{2} p_{3} p_{5}\right)^{n}+\left(p_{2} p_{4} p_{5}\right)^{n}+\left(p_{3} p_{4} p_{5}\right)^{n} \\
P_{I V}(n) & =\left(p_{1} p_{2} p_{3} p_{4}\right)^{n}+\left(p_{1} p_{2} p_{3} p_{5}\right)^{n}+\left(p_{1} p_{2} p_{4} p_{5}\right)^{n}+\left(p_{1} p_{3} p_{4} p_{5}\right)^{n}+\left(p_{2} p_{3} p_{4} p_{5}\right)^{n} \\
P_{V}(n) & =\left(p_{1} p_{2} p_{3} p_{4} p_{5}\right)^{n}=k^{n}
\end{aligned}
$$

The relationships between Pentanacci sequences of I, II, III, IV and V classes of quintic polynomial hold (6), (7).

$$
\begin{aligned}
& P_{I}(-n)=\frac{1}{p^{n}}+\frac{1}{p_{2}^{n}}+\frac{1}{p_{3}^{n}}+\frac{1}{p_{4}^{n}}+\frac{1}{p_{5}^{n}}= \\
= & \frac{\left(p_{1} p_{2} p_{3} p_{4}\right)^{n}+\left(p_{1} p_{2} p_{3} p_{5}\right)^{n}+\left(p_{1} p_{2} p_{4} p_{5}\right)^{n}+\left(p_{1} p_{3} p_{4} p_{5}\right)^{n}+\left(p_{2} p_{3} p_{4} p_{5}\right)^{n}}{\left(p_{1} p_{2} p_{3} p_{4} p_{5}\right)^{n}}=\frac{P_{I V}(n)}{P_{V}(n)} \\
P_{I I}(-n)= & \frac{1}{\left(p_{1} p_{2}\right)^{n}}+\frac{1}{\left(p_{1} p_{3}\right)^{n}}+\ldots+\frac{1}{\left(p_{4} p_{5}\right)^{n}}=\frac{\left(p_{1} p_{2} p_{3}\right)^{n}+\ldots+\left(p_{3} p_{4} p_{5}\right)^{n}}{\left(p_{1} p_{2} p_{3} p_{4} p_{5}\right)^{n}}=\frac{P_{I I I}(n)}{P_{V}(n)}
\end{aligned}
$$

As $P_{V}(n)=k_{0}{ }^{n}$, it follows that (9) and (10), as a new form of Newton-Girard formulae.

$$
\begin{gathered}
P_{I}(-n)=\frac{P_{I V}(n)}{\left(k_{0}\right)^{n}}, \quad P_{I I}(-n)=\frac{P_{I I I}(n)}{\left(k_{0}\right)^{n}} \\
\left(k_{0}\right)^{n}=\frac{P_{I V}(n)}{P_{I}(-n)}=\frac{P_{I I I}(n)}{P_{I I}(-n)}
\end{gathered}
$$

\subsection{Newton's Identities of Pentanacci Sequence}

Let us consider the relationship between I and II classes of Pentanacci sequence. We begin our research by analyzing Newton's identities for the quintic polynomial (10):

$$
x^{n+5}=k_{4} x^{n+4}+k_{3} x^{n+3}+k_{2} x^{n+2}+k_{1} x^{n+1}+k_{0} x^{n}
$$

Initial numbers of Pentanacci sequence of the first class are obtained by Albert Girard's Formulae (11):

$$
\begin{aligned}
& P_{I}(0)=I_{0}=5, P_{I}(1)=I_{1}=k_{4}, P_{I}(2)=I_{2}=k_{4}^{2}+2 k_{3} \\
& P_{I}(3)=I_{3}=k_{4}^{3}+3 k_{4} k_{3}+3 k_{2}, P_{I}(4)=I_{4}=k_{4}^{4}+4 k_{4}^{2} k_{3}+2 k_{3}^{2}+4 k_{4} k_{2}+4 k_{1}
\end{aligned}
$$

Based on the quintic polynomial (11), values of I class of Pentanacci sequence are obtained (12):

$$
\left.\begin{array}{l}
P_{I}(5)=k_{4} I_{4}+k_{3} I_{3}+k_{2} I_{2}+k_{1} I_{1}+k_{0} I_{0} \\
P_{I}(6)=k_{4} P_{I}(5)+k_{3} I_{4}+k_{2} I_{5}+k_{1} I_{2}+k_{0} I_{1} \\
P_{I}(7)=k_{4} P_{I}(6)+k_{3} P_{I}(5)+k_{2} I_{4}+k_{1} I_{3}+k_{0} I_{2} \\
\ldots \\
\ldots \\
P_{I}(n)=k_{4} P_{I}(n-1)+k_{3} P_{I}(n-2)+k_{2} P_{I}(n-3)+k_{1} P_{I}(n-4)+k_{0} P_{I}(n-5)
\end{array}\right\}
$$


For negative integers, (13) holds:

$$
\left.\begin{array}{l}
P_{I}(-1)=\frac{I_{4}-k_{4} I_{3}-k_{3} I_{2}-k_{2} I_{1}-k_{1} I_{0}}{k_{0}} \\
P_{I}(-2)=\frac{I_{3}-k_{4} I_{2}-k_{3} I_{1}-k_{2} I_{0}-k_{1} P_{I}(-1)}{k_{0}} \\
P_{I}(-3)=\frac{I_{2}-k_{4} I_{1}-k_{3} I_{0}-k_{2} P_{I}(-1)-k_{1} P_{I}(-2)}{k_{0}} \\
\ldots \\
\cdots \\
P_{I}(-n-5)=\frac{P_{I}(-n)-k_{4} P_{I}(-n-1)-k_{3} P_{I}(-n-2)-k_{2} P_{I}(-n-3)-k_{1} P_{I}(-n-4)}{k_{0}}
\end{array}\right\}
$$

Pentanacci sequences of first and second class are given by (14):

$$
\begin{aligned}
P_{I}(n) & =p_{1}^{n}+p_{2}^{n}+p_{3}^{n}+p_{4}^{n}+p_{5}^{n} \\
P_{I I}(n) & =\left(p_{1} p_{2}\right)^{n}+\left(p_{1} p_{3}\right)^{n}+\left(p_{1} p_{4}\right)^{n}+\left(p_{1} p_{5}\right)^{n}+\left(p_{2} p_{3}\right)^{n} \\
& +\left(p_{2} p_{4}\right)^{n}+\left(p_{2} p_{5}\right)^{n}+\left(p_{3} p_{4}\right)^{n}+\left(p_{3} p_{5}\right)^{n}+\left(p_{4} p_{5}\right)^{n}
\end{aligned}
$$

The relationship between Pentanacci sequences of second classes is presented with (15):

$$
P_{I I}(n)=p_{1}^{n}\left(p_{2}^{n}+p_{3}^{n}+p_{4}^{n}+p_{5}^{n}\right)+p_{2}^{n}\left(p_{3}^{n}+p_{4}^{n}+p_{5}^{n}\right)+p_{3}^{n}\left(p_{4}^{n}+p_{5}^{n}\right)+p_{4}^{n}\left(p_{5}^{n}\right)
$$

Subtracting (14) in (15), we get (16):

$$
\begin{aligned}
P_{I I}(n)= & p_{1}^{n}\left(P_{I}(n)-p_{1}^{n}\right)+p_{2}^{n}\left(P_{I}(n)-p_{1}^{n}-p_{2}^{n}\right)+p_{3}^{n}\left(P_{I}(n)-p_{1}^{n}-p_{2}^{n}-p_{3}^{n}\right) \\
& +p_{4}^{n}\left(P_{I}(n)-p_{1}^{n}-p_{2}^{n}-p_{3}^{n}-p_{4}^{n}\right) \\
P_{I I}(n)= & p_{1}^{n} P_{I}(n)-p_{1}^{2 n}+p_{2}^{n} P_{I}(2)-p_{1}^{n} p_{2}^{n}-p_{2}^{2 n}+p_{3}^{n} P_{I}(n)-p_{1}^{n} p_{3}^{n}-p_{2}^{n} p_{3}^{n}-p_{3}^{2 n} \\
& +p_{4}^{n} P_{I}(n)-p_{1}^{n} p_{4}^{n}-p_{2}^{n} p_{4}^{n}-p_{3}^{n} p_{4}^{n}-p_{4}^{2 n}
\end{aligned}
$$

In this way, we obtain a suitable form for expressing Pentanacci sequences of second class (17):

$$
\begin{aligned}
& P_{I I}(n)=\quad P_{I}(n) \underbrace{\left(p_{1}^{n}+p_{2}^{n}+p_{3}^{n}+p_{4}^{n}\right)}_{P_{I}(n)-p_{5}^{n}} \underbrace{-p_{1}^{2 n}-p_{2}^{2 n}-p_{3}^{2 n}-p_{4}^{2 n}}_{p_{5}^{2 n}-P_{I}(2 n)} \\
& \underbrace{\left.-p_{1}^{n} p_{2}^{n}-p_{1}^{n} p_{3}^{n}-p_{2}^{n}+p_{3}^{n}-p_{2}^{n}+p_{4}^{n}-p_{3}^{2 n}+p_{4}^{2 n}\right)}_{+p_{5}^{2 n}}-P_{I I}^{n} \frac{p_{I}^{n}}{(2 n)}-\frac{p_{3}^{n} p_{4}^{n}}{P_{I}(2 n)-p_{5}^{2 n}}
\end{aligned}
$$

Furthermore, we get (18).

$$
2 P_{I I}(n)=P_{I}(n)\left(P_{I}(n)-p_{5}^{n}\right)+p_{5}^{2 n}-P_{I}(2 n)+p_{5}^{n}\left(P_{I}(n)-p_{5}^{n}\right)=P_{I}^{2}(n)-P_{I}(2 n)
$$

A Pentanacci sequence of second class is, in fact, a function of a Pentanacci sequence of first class and can be expressed in the following way (19):

$$
P_{I I}(n)=\frac{P_{I}^{2}(n)-P_{I}(2 n)}{2}
$$

The same procedure for a Pentanacci sequence of third class is extremely robust (1250 members and 4 consequent calibrations of 300 members that results by total processing of 2450 members). We get expressions for Pentanacci sequences of III class as a function of Pentanacci sequences of I and II class (20):

$$
33 P_{I I I}(n)=-14 P_{I}^{3}(n)+39 P_{I}(n) P_{I I}(n)+3 P_{I}(n) P_{I}(2 n)+11 P_{I}(3 n)
$$


Replacing $P_{I I}(n)$ from (20), we get the expression for Pentanacci sequence of third class as a function of Pentanacci sequence of first class (21):

$$
P_{I I I}(n)=\frac{P_{I}^{3}(n)-3 P_{I}(n) P_{I}(2 n)+2 P_{I}(3 n)}{6}
$$

A simplified form for the Pentanacci sequence of third class is obtained from (9), a new form of Newton-Girard formula. Thus, it follows that (22):

$$
P_{I I}(-n)=\frac{P_{I I I}(n)}{P_{V}(n)} \Leftrightarrow P_{I I I}(n)=P_{I I}(-n) P_{V}(n)=P_{V}(n) \frac{P_{I}^{2}(-n)-P_{I}(-2 n)}{2}
$$

The fourth class of Pentanacci sequence is extremely robust for processing. Therefore, using (9) we get (23), which is an incomparably easier way to calculate a Pentanacci sequence of fourth class:

$$
P_{I}(-n)=\frac{P_{I V}(n)}{P_{V}(n)} \Leftrightarrow P_{I V}(n)=P_{I}(-n) P_{V}(n)
$$

The Pentanacci sequence of fifth class is the simplest to calculate.

$$
P_{V}(n)=k_{0}^{n}
$$

\section{Roots of the Quintic Polynomial}

The quintic polynomial has at least one real root. Thus, there are quintic polynomials with all real roots, three real roots and one conjugated complex root, one real root, and two conjugated complex roots. Let us consider all the possible cases.

\subsection{Quintic Polynomial with All Real Roots}

If all roots $p_{1}, p_{2}, p_{3}, p_{4}, p_{5}$ of quintic polynomial are real, the following limits exist (25):

$$
\begin{gathered}
\lim _{n \rightarrow+\infty} \frac{P_{I}(n+1)}{P_{I}(n)}=p_{1}, \quad \lim _{n \rightarrow+\infty} \frac{P_{I I}(n+1)}{P_{I I}(n)}=p_{1} p_{2}, \quad \lim _{n \rightarrow+\infty} \frac{P_{I I I}(n+1)}{P_{I I I}(n)}=p_{1} p_{2} p_{3}, \\
\lim _{n \rightarrow+\infty} \frac{P_{I V}(n+1)}{P_{I V}(n)}=p_{1} p_{2} p_{3} p_{4}, \quad \lim _{n \rightarrow \infty} \frac{P_{V}(n+1)}{P_{V}(n)}=p_{1} p_{2} p_{3} p_{4} p_{5}
\end{gathered}
$$

In the following theorem, we shall prove that the existence of the limits (25) depends on the relationship between the values of the roots of the quintic polynomial.

Theorem 1. Let

$$
x^{5}+P_{I}(1) x^{4}+P_{I I}(1) x^{3}+P_{I I I}(1) x^{2}+P_{I V}(1) x+P_{V}(1)=0
$$

be the quintic polynomial with all real roots $p_{1}, p_{2}, p_{3}, p_{4}, p_{5}$. If:

$$
\left|p_{1}\right|>\left|p_{2}\right|>\left|p_{3}\right|>\left|p_{4}\right|>\left|p_{5}\right|
$$

holds, the inequality (28) holds, too:

$$
\begin{gathered}
\lim _{n \rightarrow+\infty} \frac{P_{I}(n+1)}{P_{I}(n)}=p_{1}, \frac{\lim _{n \rightarrow+\infty} \frac{P_{I I}(n+1)}{P_{I I}(n)}}{\lim _{n \rightarrow+\infty} \frac{P_{I}(n+1)}{P_{I}(n)}}=p_{2}, \frac{\lim _{n \rightarrow+\infty} \frac{P_{I I I}(n+1)}{P_{I I I}(n)}}{\lim _{n \rightarrow+\infty} \frac{P_{I I}(n+1)}{P_{I I}(n)}}=p_{3}, \\
\frac{\lim _{n \rightarrow+\infty} \frac{P_{I V}(n+1)}{P_{I V}(n)}}{\lim _{n \rightarrow+\infty} \frac{P_{I I I}(n+1)}{P_{I I I}(n)}}=p_{4}, \frac{\lim _{n \rightarrow \infty} \frac{P_{V}(n+1)}{P_{V}(n)}}{\lim _{n \rightarrow+\infty} \frac{P_{I V}(n+1)}{P_{I V}(n)}}=p_{5}
\end{gathered}
$$


Proof. It is obvious that:

$$
\lim _{n \rightarrow+\infty} \frac{P_{I}(n+1)}{P_{I}(n)}=\lim _{n \rightarrow+\infty} \frac{p_{1}^{n+1}+p_{2}^{n+1}+p_{3}^{n+1}+p_{4}^{n+1}+p_{5}^{n+1}}{p_{1}^{n}+p_{2}^{n}+p_{3}^{n}+p_{4}^{n}+p_{5}^{n}}
$$

Further, it follows that:

$$
\lim _{n \rightarrow+\infty} \frac{P_{I}(n+1)}{P_{I}(n)}=\frac{p_{1}^{n+1}}{p_{1}^{n}} \lim _{n \rightarrow+\infty} \frac{1+\frac{p_{2}^{n+1}}{p_{1}^{n+1}}+\frac{p_{3}^{n+1}}{p_{1}^{n+1}}+\frac{p_{4}^{n+1}}{p_{1}^{n+1}}+\frac{p_{5}^{n+1}}{p_{1}^{n+1}}}{1+\frac{p_{2}^{n}}{p_{1}^{n}}+\frac{p_{3}^{n}}{p_{1}^{n}}+\frac{p_{4}^{n}}{p_{1}^{n}}+\frac{p_{5}^{n}}{p_{1}^{n}}}
$$

From (28), it follows that:

$$
\lim _{n \rightarrow+\infty} \frac{p_{i \neq 1}^{n}}{p_{1}^{n}}=\lim _{n \rightarrow+\infty} \frac{p_{i \neq 1}^{n+1}}{p_{1}^{n+1}}=0
$$

Finally, we get (32):

$$
\lim _{n \rightarrow+\infty} \frac{p_{1}^{n+1}}{p_{1}^{n}} \cdot \frac{1+\frac{p_{2}^{n+1}}{p_{1}^{n+1}}+\frac{p_{3}^{n+1}}{p_{1}^{n+1}}+\frac{p_{4}^{n+1}}{p_{1}^{n+1}}+\frac{p_{5}^{n+1}}{p_{1}^{n+1}}}{1+\frac{p_{2}^{n}}{p_{1}^{n}}+\frac{p_{3}^{n}}{p_{1}^{n}}+\frac{p_{4}^{n}}{p_{1}^{n}}+\frac{p_{5}^{n}}{p_{1}^{n}}}=p_{1} \lim _{n \rightarrow+\infty} \frac{1+0+0+0+0}{1+0+0+0+0}=p_{1}
$$

The second equation from (28) is proven analogously:

$$
\begin{aligned}
& \begin{array}{l}
\lim _{n \rightarrow+\infty} \frac{P_{I I}(n+1)}{P_{I}(n)} \\
\lim _{n \rightarrow+\infty} \frac{P_{I}(n+1)}{P_{I}(n)}
\end{array}=\lim _{n \rightarrow+\infty} \frac{\left(p_{1} p_{2}\right)^{n+1}+\left(p_{1} p_{3}\right)^{n+1}+\cdots+\left(p_{4} p_{5}\right)^{n+1}}{p_{1}\left(p_{1} p_{2}\right)^{n}+p_{1}\left(p_{1} p_{3}\right)^{n}+\cdots+p_{1}\left(p_{4} p_{5}\right)^{n}}= \\
& =\underbrace{\lim _{n \rightarrow+\infty} \frac{p_{1}^{n+1} p_{2}^{n+1}}{p_{1}^{n+1} p_{2}^{n}+p_{1}^{n+1} p_{3}^{n}+\ldots+p_{1}\left(p_{4} p_{5}\right)^{n}}}_{=p_{2}}+\underbrace{\lim _{n \rightarrow+\infty} \frac{p_{1}^{n+1} p_{3}^{n+1}}{p_{1}^{n+1} p_{2}^{n}+p_{1}^{n+1} p_{3}^{n}+\ldots+p_{1}\left(p_{4} p_{5}\right)^{n}}}_{=0}+\ldots \\
& \ldots+\underbrace{\lim _{n \rightarrow+\infty} \frac{p_{3}^{n+1} p_{5}^{n+1}}{p_{1}^{n+1} p_{2}^{n}+p_{1}^{n+1} p_{3}^{n}+\ldots+p_{1}\left(p_{4} p_{5}\right)^{n}}}_{=0}+\underbrace{\lim _{n \rightarrow+\infty} \frac{p_{4}^{n+1} p_{5}^{n+1}}{p_{1}^{n+1} p_{2}^{n}+p_{1}^{n+1} p_{3}^{n}+\ldots+p_{1}\left(p_{4} p_{5}\right)^{n}}}_{=0}=p_{2}
\end{aligned}
$$

The third equation from (28) is proven in the following way:

$$
\frac{\lim _{n \rightarrow+\infty} \frac{P_{I I I}(n+1)}{P_{I I I}(n)}}{\lim _{n \rightarrow+\infty} \frac{P_{I I}(n+1)}{P_{I I}(n)}}=\lim _{n \rightarrow+\infty} \frac{\frac{\left(p_{1} p_{2} p_{3}\right)^{n+1}+\left(p_{1} p_{2} p_{4}\right)^{n+1}+\cdots+\left(p_{3} p_{4} p_{5}\right)^{n+1}}{\left(p_{1} p_{2} p_{3}\right)^{n}+\left(p_{1} p_{2} p_{4}\right)^{n}+\cdots+\left(p_{3} p_{4} p_{5}\right)^{n}}}{\frac{\left(p_{1} p_{2}\right)^{n+1}+\left(p_{1} p_{3}\right)^{n+1}+\cdots+\left(p_{4} p_{5}\right)^{n+1}}{\left(p_{1} p_{2}\right)^{n}+\left(p_{1} p_{3}\right)^{n}+\cdots+\left(p_{4} p_{5}\right)^{n}}}
$$

Further, it follows that:

$$
\frac{\lim _{n \rightarrow+\infty} \frac{P_{I I}(n+1)}{P_{I I}(n)}}{\lim _{n \rightarrow+\infty} \frac{P_{I}(n+1)}{P_{I}(n)}}=p_{2} \Leftrightarrow \lim _{n \rightarrow+\infty} \frac{P_{I I}(n+1)}{P_{I I}(n)}=p_{2} \lim _{n \rightarrow+\infty} \frac{P_{I}(n+1)}{P_{I}(n)}=p_{1} p_{2}
$$

Subtracting (35) in (34), we get:

$$
\frac{\lim _{n \rightarrow+\infty} \frac{P_{I I I}(n+1)}{P_{I I I}(n)}}{\lim _{n \rightarrow+\infty} \frac{P_{I I}(n+1)}{P_{I I}(n)}}=\lim _{n \rightarrow+\infty} \frac{1}{p_{1} p_{2}} \cdot \frac{\left(p_{1} p_{2} p_{3}\right)^{n+1}+\left(p_{1} p_{2} p_{4}\right)^{n+1}+\cdots+\left(p_{3} p_{4} p_{5}\right)^{n+1}}{\left(p_{1} p_{2} p_{3}\right)^{n}+\left(p_{1} p_{2} p_{4}\right)^{n}+\cdots+\left(p_{3} p_{4} p_{5}\right)^{n}}
$$


As $\left|\left(p_{1} p_{2} p_{3}\right)^{n+1}\right|$ is larger than the absolute value of each other addend in the denominator in (36), it follows that:

$$
\underbrace{\lim _{n \rightarrow+\infty} \frac{\left(p_{1} p_{2} p_{3}\right)^{n+1}}{p_{1}^{n+1} p_{2}^{n+1} p_{3}^{n}+p_{1}^{n+1} p_{2}^{n+1} p_{4}^{n}+\cdots+p_{1} p_{2} p_{3}^{n} p_{4}^{n} p_{5}^{n}}}_{p_{3}}+0+\ldots+0=p_{3}
$$

Analogously, we get:

$$
\begin{gathered}
\lim _{n \rightarrow+\infty} \frac{P_{I I I}(n+1)}{P_{I I I}(n)}=p_{3} \lim _{n \rightarrow+\infty} \frac{P_{I I}(n+1)}{P_{I I}(n)}=p_{1} p_{2} p_{3} \\
\frac{\lim _{n \rightarrow+\infty} \frac{P_{I V}(n+1)}{P_{I V}(n)}}{\lim _{n \rightarrow+\infty} \frac{P_{I I I}(n+1)}{P_{I I I}(n)}}=\lim _{n \rightarrow+\infty} \frac{1}{p_{1} p_{2} p_{3}} \cdot \frac{\left(p_{1} p_{2} p_{3} p_{4}\right)^{n+1}+\cdots+\left(p_{2} p_{3} p_{4} p_{5}\right)^{n+1}}{\left(p_{1} p_{2} p_{3} p_{4}\right)^{n}+\cdots+\left(p_{2} p_{3} p_{4} p_{5}\right)^{n}} \\
\lim _{n \rightarrow+\infty} \frac{\left(p_{1} p_{2} p_{3} p_{4}\right)^{n+1}}{\left(p_{1} p_{2} p_{3}\right)^{n+1} p_{4}^{n}+\left(p_{1} p_{2} p_{3}\right)^{n+1} p_{4}^{n}+\cdots+p_{1}\left(p_{2} p_{3}\right)^{n+1}\left(p_{4} p_{5}\right)^{n+1}}+0+\ldots+0=p_{4} \\
\lim _{n \rightarrow+\infty} \frac{P_{I V}(n+1)}{P_{I V}(n)}=p_{3} \lim _{n \rightarrow+\infty} \frac{P_{I I I}(n+1)}{P_{I I I}(n)}=p_{1} p_{2} p_{3} p_{4} \\
\frac{\lim _{n \rightarrow+\infty} \frac{P_{V}(n+1)}{P_{V}(n)}}{\lim _{n \rightarrow+\infty} \frac{P_{I V}(n+1)}{P_{I V}(n)}}=\lim _{n \rightarrow+\infty} \frac{1}{p_{1} p_{2} p_{3} p_{4}} \cdot \frac{\left(p_{1} p_{2} p_{3} p_{4} p_{5}\right)^{n+1}}{\left(p_{1} p_{2} p_{3} p_{4} p_{5}\right)^{n}}=p_{5}
\end{gathered}
$$

Note: The relationship between the absolute values of the roots $p_{1}, p_{2}, p_{3}, p_{4}, p_{5}$ of the quintic polynomial given by (27) induces the order of the values of the limits that are calculated in (28).

From (28) for $n \rightarrow-\infty$, the following holds:

$$
\begin{gathered}
\lim _{n \rightarrow-\infty} \frac{P_{I}(n-1)}{P_{I}(n)}=\frac{1}{p_{5}}, \frac{\lim _{n \rightarrow-\infty} \frac{P_{I I}(n-1)}{P_{I I}(n)}}{\lim _{n \rightarrow-\infty} \frac{P_{I}(n-1)}{P_{I}(n)}}=\frac{1}{p_{4}}, \frac{\lim _{n \rightarrow-\infty} \frac{P_{I I I}(n-1)}{P_{I I I}(n)}}{\lim _{n \rightarrow-\infty} \frac{P_{I I}(n-1)}{P_{I I}(n)}}=\frac{1}{p_{3}} \\
\frac{\lim _{n \rightarrow-\infty} \frac{P_{I V}(n-1)}{P_{I V}(n)}}{\lim _{n \rightarrow-\infty} \frac{P_{I I I}(n-1)}{P_{I I I}(n)}}=\frac{1}{p_{2}}, \frac{\lim _{n \rightarrow-\infty} \frac{P_{V}(n-1)}{P_{V}(n)}}{\lim _{n \rightarrow-\infty} \frac{P_{I V}(n-1)}{P_{I V}(n)}}=\frac{1}{p_{1}}
\end{gathered}
$$

The Proof for (43) is analogous to the Proof for (29).

Equations (28) and (43) play a key role for finding the complex roots of the quintic polynomial. In the case of complex solutions, the values of the limits oscillate. These oscillations can be explained by the trigonometric form of the complex number. However, we withdraw the trigonometric attitude because the aim of the model is the application of mathematical radicals and limits of sequences.

Consecutive products of conjugated complex roots can be expressed by the square of the modulus. The theorem can easily be proven for complex solutions, which will be evident in Sections 3.2 and 3.3.

\subsection{Quintic Polynomials with Three Real Roots and One Conjugated Complex Root}

We denote by $p_{1}, p_{2}$, and $p_{3}$ as three real roots of the quintic polynomial. We denote by $z_{1,2}$ conjugate complex solutions of the polynomial, with $\operatorname{Re}\left(z_{1,2}\right)=a, \operatorname{Im}\left(z_{1,2}\right)=b$ and $\left|z_{1,2}\right|=a^{2}+b^{2}=\rho^{2}$.

In the case that one conjugate complex root of the polynomial exists, one of the five sequences does not converge. Which sequence does not converge depends on the relationship between the absolute values of real roots and moduli. The Pentanacci sequence of the fifth class always converges. There are four cases:

- $\left|p_{1}\right|>\left|p_{2}\right|>\left|p_{3}\right|>\rho^{2}$ 
- $\left|p_{1}\right|>\left|p_{2}\right|>\rho^{2}>\left|p_{3}\right|$

- $\left|p_{1}\right|>\rho^{2}>\left|p_{2}\right|>\left|p_{3}\right|$

- $\rho^{2}>\left|p_{1}\right|>\left|p_{2}\right|>\left|p_{3}\right|$

Let us consider all the possible cases.

3.2.1. $\left|p_{1}\right|>\left|p_{2}\right|>\left|p_{3}\right|>\rho^{2}$

It is obvious that:

$$
\lim _{n \rightarrow+\infty} \frac{P_{I}(n+1)}{P_{I}(n)}=p_{1}, \quad \lim _{n \rightarrow+\infty} \frac{P_{I I}(n+1)}{P_{I I}(n)}=p_{1} p_{2}, \quad \lim _{n \rightarrow+\infty} \frac{P_{I I I}(n+1)}{P_{I I I}(n)}=p_{1} p_{2} p_{3}
$$

However, the limit of Pentanacci sequence of the fourth class does not exist, i.e.,

$$
\lim _{n \rightarrow+\infty} \frac{P_{I V}(n+1)}{P_{I V}(n)} \neq \text { const }
$$

For the Pentanacci sequence of the fifth class, the following always holds:

$$
\lim _{n \rightarrow+\infty} \frac{P_{V}(n+1)}{P_{V}(n)}=\lim _{n \rightarrow+\infty} \frac{\left(p_{1} p_{2} p_{3}(a+i b)(a-i b)\right)^{n+1}}{\left(p_{1} p_{2} p_{3}(a+i b)(a-i b)\right)^{n}}=p_{1} p_{2} p_{3}\left(a^{2}+b^{2}\right)=p_{1} p_{2} p_{3} \rho^{2}
$$

3.2.2. $\left|p_{1}\right|>\left|p_{2}\right|>\rho^{2}>\left|p_{3}\right|$

It is obvious that:

$$
\lim _{n \rightarrow+\infty} \frac{P_{I}(n+1)}{P_{I}(n)}=p_{1}, \quad \lim _{n \rightarrow+\infty} \frac{P_{I I}(n+1)}{P_{I I}(n)}=p_{1} p_{2}
$$

However, the limit of Pentanacci sequence of the third class does not exist, i.e.,

$$
\lim _{n \rightarrow+\infty} \frac{P_{I I I}(n+1)}{P_{I I I}(n)} \neq \text { const }
$$

The Pentanacci sequence of the fourth class converges to:

$$
\lim _{n \rightarrow+\infty} \frac{P_{I V}(n+1)}{P_{I V}(n)}=p_{1} p_{2} \rho^{2}
$$

For the Pentanacci sequence of the fifth class, the following always holds:

$$
\lim _{n \rightarrow+\infty} \frac{P_{V}(n+1)}{P_{V}(n)}=p_{1} p_{2} p_{3} \rho^{2}
$$

3.2.3. $\left|p_{1}\right|>\rho^{2}>\left|p_{2}\right|>\left|p_{3}\right|$

It is obvious that:

$$
\lim _{n \rightarrow+\infty} \frac{P_{I}(n+1)}{P_{I}(n)}=p_{1}
$$

However, the limit of Pentanacci sequence of the second class does not exist, i.e.,

$$
\lim _{n \rightarrow+\infty} \frac{P_{I I}(n+1)}{P_{I I}(n)} \neq \text { const }
$$


Pentanacci sequences of the third and the fourth class converge to:

$$
\lim _{n \rightarrow+\infty} \frac{P_{I I I}(n+1)}{P_{I I I}(n)}=p_{1} \rho^{2}, \quad \lim _{n \rightarrow+\infty} \frac{P_{I V}(n+1)}{P_{I V}(n)}=p_{1} p_{2} \rho^{2},
$$

For the Pentanacci sequence of the fifth class, the following always holds:

$$
\lim _{n \rightarrow+\infty} \frac{P_{V}(n+1)}{P_{V}(n)}=p_{1} p_{2} p_{3} \rho^{2}
$$

3.2.4. $\rho^{2}>\left|p_{1}\right|>\left|p_{2}\right|>\left|p_{3}\right|$

In this case, the limit of Pentanacci sequence of the first class does not exist, i.e.,

$$
\lim _{n \rightarrow+\infty} \frac{P_{I}(n+1)}{P_{I}(n)} \neq \text { const }
$$

Pentanacci sequences of the second class converge to:

$$
\lim _{n \rightarrow+\infty} \frac{P_{I I}(n+1)}{P_{I I}(n)}=\rho^{2}
$$

It is obvious that:

$$
\begin{gathered}
\lim _{n \rightarrow+\infty} \frac{P_{I I I}(n+1)}{P_{I I I}(n)}=\rho^{2} p_{1}, \quad \lim _{n \rightarrow+\infty} \frac{P_{I V}(n+1)}{P_{I V}(n)}=\rho^{2} p_{1} p_{2}, \\
\lim _{n \rightarrow+\infty} \frac{P_{V}(n+1)}{P_{V}(n)}=\rho^{2} p_{1} p_{2} p_{3}
\end{gathered}
$$

3.2.5. Values $\operatorname{Re}(z)=a$ and $\operatorname{Im}(z)=b$

The convergence of Pentanacci sequences induces the character of the roots of the quintic polynomial in the sense of existence of conjugated complex roots. In Section 3.2, one of the Pentanacci sequences does not converge. Let us introduce the procedure for calculating the real roots $p_{1}, p_{2}$, and $p_{3}$ of the quintic polynomial:

- If the fourth class does not converge, then the real solutions are directly obtained from (46);

- If the third class does not converge (48), then the real solutions are directly obtained from (47); (49) and (50);

- If the second class does not converge (52), then the real solutions are directly obtained from (51), (53) and (54);

- If the first class does not converge (56), then the real solutions are directly obtained from (56) and (57).

From the Pentanacci sequence of the first class, for the initial number $I_{1}=P_{I}(1)(11)$ and the square of modulus, the values $a$ and $b$ are calculated as follows:

$$
\begin{aligned}
P_{I}(1)=p_{1}+p_{2}+p_{3}+(a+i b)+(a-i b) \Leftrightarrow \quad a & =\frac{P_{I}(1)-p_{1}-p_{2}-p_{3}}{2} \\
b & =\sqrt{\rho^{2}-\frac{\left(P_{I}(1)-p_{1}-p_{2}-p_{3}\right)^{2}}{4}}
\end{aligned}
$$

\subsection{Quintic Polynomial with One Real Root and Two Conjugated Complex Roots}

Denote by $p$ the real root and by $z_{1,2}$ and $z_{3,4}$ conjugate complex roots of the quintic polynomial, with $\operatorname{Re}\left(z_{1,2}\right)=a_{1}, \operatorname{Im}\left(z_{1,2}\right)=b_{1}, \operatorname{Re}\left(z_{3,4}\right)=a_{2}, \operatorname{Im}\left(z_{3,4}\right)=b_{2},\left|z_{1,2}\right|=a_{1}{ }^{2}+b_{1}{ }^{2}=\rho_{1}{ }^{2}$ and $\left|z_{3,4}\right|=a_{2}{ }^{2}+b_{2}{ }^{2}=\rho_{2}{ }^{2}$. Without the loss of generality, we can assume that $\rho_{1}>\rho_{2}$. Let us consider all the possible cases: 
- $|p|>\left(\rho_{1}\right)^{2}>\left(\rho_{2}\right)^{2}$

- $\quad\left(\rho_{1}\right)^{2}>|p|>\left(\rho_{2}\right)^{2}$

- $\left(\rho_{1}\right)^{2}>\left(\rho_{2}\right)^{2}>|p|$

3.3.1. $|p|>\left(\rho_{1}\right)^{2}>\left(\rho_{2}\right)^{2}$

It is obvious that:

$$
\lim _{n \rightarrow+\infty} \frac{P_{I}(n+1)}{P_{I}(n)}=p, \quad \lim _{n \rightarrow+\infty} \frac{P_{I I I}(n+1)}{P_{I I I}(n)}=p \rho_{1}^{2}, \quad \lim _{n \rightarrow+\infty} \frac{P_{V}(n+1)}{P_{V}(n)}=p \rho_{1}^{2} \rho_{2}^{2}
$$

The second and the fourth classes of Pentanacci sequences do not converge, i.e.,

$$
\lim _{n \rightarrow+\infty} \frac{P_{I I}(n+1)}{P_{I I}(n)} \neq \text { const, } \quad \lim _{n \rightarrow+\infty} \frac{P_{I V}(n+1)}{P_{I V}(n)} \neq \text { const },
$$

Further, we obtain:

$$
\frac{\lim _{n \rightarrow+\infty} \frac{P_{I I I}(n+1)}{P_{I I I}(n)}}{\lim _{n \rightarrow+\infty} \frac{P_{I}(n+1)}{P_{I}(n)}}=\rho_{1}^{2}, \quad \frac{\lim _{n \rightarrow+\infty} \frac{P_{V}(n+1)}{P_{V}(n)}}{\lim _{n \rightarrow+\infty} \frac{P_{I I I}(n+1)}{P_{I I I}(n)}}=\rho_{2}^{2}
$$

3.3.2. $\left(\rho_{1}\right)^{2}>|p|>\left(\rho_{2}\right)^{2}$

In this case, it is obvious that:

$$
\lim _{n \rightarrow \pm \infty} \frac{P_{I}(n \pm 1)}{P_{I}(n)} \neq \text { const }
$$

In this case,

$$
\lim _{n \rightarrow+\infty} \frac{P_{I I}(n+1)}{P_{I I}(n)}=\rho_{1}^{2}
$$

holds. In this case, there are two possibilities:

If $|p|$ is closer to $\left(\rho_{1}\right)^{2}$, then:

$$
\lim _{n \rightarrow+\infty} \frac{P_{I I I}(n+1)}{P_{I I I}(n)}=\rho_{1}^{2} p, \quad \lim _{n \rightarrow+\infty} \frac{P_{I V}(n+1)}{P_{I V}(n)} \neq \text { const, } \quad \frac{\lim _{n \rightarrow+\infty} \frac{P_{(I I I)}(n+1)}{P_{(I I I)}(n)}}{\lim _{n \rightarrow+\infty} \frac{P_{V}(n+1)}{P_{V}(n)}}=\rho_{1}^{2} p \rho_{2}^{2},
$$

holds. If $|p|$ is closer to $\left(\rho_{2}\right)^{2}$, then

$$
\lim _{n \rightarrow+\infty} \frac{P_{I I I}(n+1)}{P_{I I I}(n)} \neq \text { const, } \quad \lim _{n \rightarrow+\infty} \frac{P_{I V}(n+1)}{P_{I V}(n)}=\rho_{1}^{2} p, \quad \frac{\lim _{n \rightarrow+\infty} \frac{P_{(I V)}(n+1)}{P_{(I V)}(n)}}{\lim _{n \rightarrow+\infty} \frac{P_{V}(n+1)}{P_{V}(n)}}=\rho_{1}^{2} p \rho_{2}^{2}
$$

holds.

3.3.3. $\left(\rho_{1}\right)^{2}>\left(\rho_{2}\right)^{2}>|p|$

In this case, (66) holds:

$$
\lim _{n \rightarrow+\infty} \frac{P_{I I}(n+1)}{P_{I I}(n)}=\rho_{1}^{2}, \quad \lim _{n \rightarrow-\infty} \frac{P_{I}(n-1)}{P_{I}(n)}=\frac{1}{p^{\prime}}, \quad \frac{\lim _{n \rightarrow+\infty} \frac{P_{V}(n+1)}{P_{V}(n)} \lim _{n \rightarrow-\infty} \frac{P_{I V}(n+1)}{P_{I V}(n)}}{\lim _{n \rightarrow+\infty} \frac{P_{I I}(n+1)}{P_{I I}(n)}}=\rho_{2}^{2}
$$


3.4. Values $\operatorname{Re}\left(z_{1,2}\right)=a_{1}, \operatorname{Im}\left(z_{1,2}\right)=b_{1}, \operatorname{Re}\left(z_{3,4}\right)=a_{2}$ and $\operatorname{Im}\left(z_{3,4}\right)=b_{2}$

The real root $p$ of the quintic polynomial is directly calculated. From the Pentanacci sequence of the first class of quintic polynomial, we obtain:

$$
\begin{aligned}
& P_{I}(1)=p+\left(a_{1}+i b_{1}\right)+\left(a_{1}-i b_{1}\right)+\left(a_{2}+i b_{2}\right)+\left(a_{2}-i b_{2}\right) \\
& P_{I}(2)=p^{2}+\left(a_{1}+i b_{1}\right)^{2}+\left(a_{1}-i b_{1}\right)^{2}+\left(a_{2}+i b_{2}\right)^{2}+\left(a_{2}-i b_{2}\right)^{2}
\end{aligned}
$$

Furthermore, we get:

$$
\begin{aligned}
& P_{I}(1)=p+2 a_{1}+2 a_{2} \Rightarrow \frac{P_{I}(1)-p}{2}=a_{1}+a_{2} \\
& P_{I}(2)=p^{2}+2 a_{1}^{2}-2 b_{1}^{2}+2 a_{2}^{2}-2 b_{2}^{2} \Rightarrow \frac{P_{I}(2)-p^{2}}{2}=a_{1}^{2}-b_{1}^{2}+a_{2}^{2}-b_{2}^{2}
\end{aligned}
$$

It is obvious that (69) holds:

$$
\rho_{1}^{2}=a_{1}^{2}+b_{1}^{2} \Rightarrow-b_{1}^{2}=a_{1}^{2}-\rho_{1}^{2}, \quad \rho_{2}^{2}=a_{2}^{2}+b_{2}^{2} \Rightarrow-b_{2}^{2}=a_{2}^{2}-\rho_{2}^{2}
$$

Values $a_{1}, a_{2}, b_{1}$ and $b_{2}$ are calculated from the system of four equations defined by (68) and (69).

$$
\begin{aligned}
& \frac{P_{I}(2)-p^{2}}{2}=a_{1}^{2}-b_{1}^{2}+a_{2}^{2}-b_{2}^{2}=a_{1}^{2}+a_{1}^{2}-\rho_{1}^{2}+a_{2}^{2}+a_{2}^{2}-\rho_{2}^{2}=2 a_{1}^{2}-\rho_{1}^{2}+2 a_{2}^{2}-\rho_{2}^{2} \\
& \frac{P_{I}(2)-p^{2}}{2}+\rho_{1}^{2}+\rho_{2}^{2}=2 a_{1}^{2}+2 a_{2}^{2} \Leftrightarrow \frac{P_{I}(2)-p^{2}+2 \rho_{1}^{2}+2 \rho_{2}^{2}}{4}=a_{1}^{2}+a_{2}^{2}
\end{aligned}
$$

Furthermore, we get:

$$
\begin{aligned}
& \frac{P_{I}(1)-p}{2}-a_{2}=a_{1} \Rightarrow a_{1}^{2}=\left(\frac{P_{I}(1)-p}{2}\right)^{2}-2 a_{2} \frac{P_{I}(1)-p}{2}+a_{2}^{2} \\
& \frac{P_{I}(2)-p^{2}+2 \rho_{1}^{2}+2 \rho_{2}^{2}}{4}=\left(\frac{P_{I}(1)-p}{2}\right)^{2}-a_{2}\left(P_{I}(1)-p\right)+a_{2}^{2}+a_{2}^{2}
\end{aligned}
$$

i.e.,

$$
\underbrace{+2}_{a} a_{2}^{2}-\underbrace{-\left(P_{I}(1)-p\right)}_{b} a_{2}+\underbrace{+\frac{P_{I}(1)^{2}-P_{I}(2)-2 P_{I}(1) p+2 p^{2}-2 \rho_{1}^{2}-2 \rho_{2}^{2}}{4}}_{c}=0
$$

It follows that:

$$
\begin{aligned}
& a_{1}=\frac{P_{I}(1)-p+\sqrt{\left(P_{I}(1)-p\right)^{2}-2\left(P_{I}(1)^{2}-P_{I}(2)-2 P_{I}(1) p+2 p^{2}-2 \rho_{1}^{2}-2 \rho_{2}^{2}\right)}}{4} \\
& a_{2}=\frac{P_{I}(1)-p-\sqrt{\left(P_{I}(1)-p\right)^{2}-2\left(P_{I}(1)^{2}-P_{I}(2)-2 P_{I}(1) p+2 p^{2}-2 \rho_{1}^{2}-2 \rho_{2}^{2}\right)}}{4}
\end{aligned}
$$

Furthermore, we get:

$$
\begin{aligned}
& b_{1}=\sqrt{\rho_{1}^{2}-\left(\frac{P_{I}(1)-p+\sqrt{\left(P_{I}(1)-p\right)^{2}-2\left(P_{I}(1)^{2}-P_{I}(2)-2 P_{I}(1) p+2 p^{2}-2 \rho_{1}^{2}-2 \rho_{2}^{2}\right)}}{4}\right)^{2}} \\
& b_{2}=\sqrt{\rho_{2}^{2}-\left(\frac{P_{I}(1)-p-\sqrt{\left(P_{I}(1)-p\right)^{2}-2\left(P_{I}(1)^{2}-P_{I}(2)-2 P_{I}(1) p+2 p^{2}-2 \rho_{1}^{2}-2 \rho_{2}^{2}\right)}}{4}\right)^{2}}
\end{aligned}
$$

\section{Numerical Examples}

We apply the New-nacci method to a polynomial:

$$
p(x)=x^{5}+0.3 x^{4}-13.29 x^{3}+2.393 x^{2}+29.064 x-17.199
$$


We start from the initial numbers of the Pentanacci sequence of the first class (11) and develop it by (12) and (13). For the Pentanacci sequences of the second, third, fourth, and fifth classes, we apply (19), (21), (23), and (24) respectively. Values are given in Table 1. Ratios of consecutive members of the Pentanacci sequences are given in Table 2.

Table 1. Pentanacci sequences of the quintic polynomial (75), $n \in[0,100]$.

\begin{tabular}{|c|c|c|c|c|c|c|}
\hline$n$ & $P_{I}(-n)$ & $P_{I}(n)$ & $P_{I I}(n)$ & $P_{I I I}(n)$ & $P_{I V}(n)$ & $P_{V}(n)$ \\
\hline 0 & $+5.00000 \times 10^{0}$ & $+5.00000 \times 10^{0}$ & $+1.00000 \times 10^{1}$ & $+1.00000 \times 10^{1}$ & $+5.00000 \times 10^{0}$ & $+1.00000 \times 10^{0}$ \\
\hline 1 & $+1.68986 \times 10^{0}$ & $-3.00000 \times 10^{1}$ & $-1.32900 \times 10^{1}$ & $-2.39300 \times 10^{0}$ & $+2.90640 \times 10^{1}$ & $+1.71990 \times 10^{1}$ \\
\hline 2 & $+3.13391 \times 10^{0}$ & $+2.66700 \times 10^{1}$ & $+2.33316 \times 10^{2}$ & $+7.67928 \times 10^{2}$ & $+9.27030 \times 10^{2}$ & $+2.95805 \times 10^{2}$ \\
\hline 3 & $+3.21286 \times 10^{0}$ & $-1.91670 \times 10^{1}$ & $-1.11942 \times 10^{3}$ & $+4.02970 \times 10^{3}$ & $+1.63456 \times 10^{4}$ & $+5.08756 \times 10^{3}$ \\
\hline 4 & $+4.62932 \times 10^{0}$ & $+2.44656 \times 10^{2}$ & $+1.53292 \times 10^{4}$ & $+1.72909 \times 10^{5}$ & $+4.05070 \times 10^{5}$ & $+8.75009 \times 10^{4}$ \\
\hline 5 & $+6.16852 \times 10^{0}$ & $-2.97233 \times 10^{2}$ & $-1.23463 \times 10^{5}$ & $+1.93680 \times 10^{6}$ & $+9.28318 \times 10^{6}$ & $+1.50492 \times 10^{6}$ \\
\hline 6 & $+8.73835 \times 10^{0}$ & $+2.60622 \times 10^{3}$ & $+1.44027 \times 10^{6}$ & $+5.26388 \times 10^{7}$ & $+2.26177 \times 10^{8}$ & $+2.58832 \times 10^{7}$ \\
\hline 7 & $+1.22859 \times 10^{1}$ & $-4.30179 \times 10^{3}$ & $-1.38383 \times 10^{7}$ & $+7.73653 \times 10^{8}$ & $+5.46931 \times 10^{9}$ & $+4.45166 \times 10^{8}$ \\
\hline$\cdots$ & $\ldots$ & $\ldots$ & $\ldots$ & $\ldots$ & $\ldots$ & $\cdots$ \\
\hline 97 & $+1.06044 \times 10^{15}$ & $-5.95113 \times 10^{52}$ & $-1.135957 \times 10^{99}$ & $+6.55835 \times 10^{123}$ & $+7.40116 \times 10^{134}$ & $+6.97927 \times 10^{119}$ \\
\hline 98 & $+1.51492 \times 10^{15}$ & $+2.08290 \times 10^{53}$ & $+1.19275 \times 10^{100}$ & $+1.23952 \times 10^{125}$ & $+1.81846 \times 10^{136}$ & $+1.20036 \times 10^{121}$ \\
\hline 99 & $+2.16418 \times 10^{15}$ & $-7.29014 \times 10^{53}$ & $-1.25239 \times 10^{101}$ & $+2.34271 \times 10^{126}$ & $+4.46797 \times 10^{137}$ & $+2.06450 \times 10^{122}$ \\
\hline 100 & $+3.09169 \times 10^{15}$ & $+2.55155 \times 10^{54}$ & $+1.31501 \times 10^{102}$ & $+4.42772 \times 10^{127}$ & $+1.09778 \times 10^{139}$ & $+3.55074 \times 10^{123}$ \\
\hline
\end{tabular}

Table 2. Ratios of consecutive members of the Pentanacci sequences for polynomial (75), $n \in[0,100]$.

\begin{tabular}{|c|c|c|c|c|c|c|}
\hline$n$ & $\frac{P_{I}(-n-1)}{P_{I}(-n)}$ & $\frac{P_{I}(n+1)}{P_{I}(n)}$ & $\frac{P_{I I}(n+1)}{P_{I I}(n)}$ & $\frac{P_{I I I}(n+1)}{P_{I I I}(n)}$ & $\frac{P_{I V}(n+1)}{P_{I V}(n)}$ & $\frac{P_{V}(n+1)}{P_{V}(n)}$ \\
\hline 0 & +0.33797314 & -0.06000000 & -1.32900000 & -0.23930000 & +5.81280000 & +17.19900000 \\
\hline 1 & +1.85453676 & -88.90000000 & -17.55577878 & -320.90604639 & +31.89617775 & +17.19900000 \\
\hline 2 & +1.02519072 & -0.71867267 & -4.79788257 & +5.24750194 & +17.63225516 & +17.19900000 \\
\hline 3 & +1.44087299 & -12.76445453 & -13.69388674 & +42.90867581 & +24.78157458 & +17.19900000 \\
\hline 4 & +1.33248699 & -1.21490201 & -8.05409883 & +11.20127397 & +22.91744371 & +17.19900000 \\
\hline 5 & +1.41660445 & -8.76826778 & -11.66561134 & +27.17822315 & +24.36417992 & +17.19900000 \\
\hline 6 & +1.40598475 & -1.65058617 & -9.60814516 & +14.69737188 & +24.18153177 & +17.19900000 \\
\hline 7 & +1.42263765 & -6.78743919 & -10.92540103 & +22.64781780 & +24.46794499 & +17.19900000 \\
\hline$\cdots$ & $\ldots$ & $\cdots$ & ... & $\ldots$ & $\ldots$ & \\
\hline 97 & +1.42857143 & -3.50000208 & -10.50000000 & +18.90000000 & +24.57000000 & +17.19900000 \\
\hline 98 & +1.42857143 & -3.49999821 & -10.50000000 & +18.90000000 & +24.57000000 & +17.19900000 \\
\hline 99 & +1.42857143 & -3.50000153 & -10.50000000 & +18.90000000 & +24.57000000 & +17.19900000 \\
\hline 100 & +1.42857143 & -3.49999869 & -10.50000000 & +18.90000000 & +24.57000000 & +17.19900000 \\
\hline
\end{tabular}

Based on the theorem, we obtain the values of the root and the product of the roots for $n=100$ : $p_{1}=-3.49999869, p_{1} p_{2}=-10.50, p_{1} p_{2} p_{3}=+18.90, p_{1} p_{2} p_{3} p_{4}=+24.57$ and $p_{1} p_{2} p_{3} p_{4} p_{5}=+17.199$. By (29), we calculate the roots of polynomial (75) for $n=100$ :

$$
\begin{aligned}
& p_{1} \approx \frac{P_{I}(100)}{P_{I}(99)}=-3.49999869 \approx-3.5, \\
& p_{2} \approx \frac{\frac{P_{I I}(100)}{P_{I I}(99)}}{\frac{P_{I}(100)}{P_{I}(99)}}=\frac{-10.5}{-3.49999869} \approx 3, p_{3} \approx \frac{\frac{P_{I I I}(100)}{P_{I I I}(99)}}{\frac{P_{I I}(100)}{P_{I I}(99)}}=\frac{+18.9}{-10.5}=-1.8, \\
& p_{4} \approx \frac{\frac{P_{I V}(100)}{P_{I V}(99)}}{\frac{P_{I I I}(100)}{P_{I I I}(99)}}=\frac{+24.57}{+18.90}=+1.3, \quad p_{5} \approx \frac{\frac{P_{V}(100)}{P_{V}(99)}}{\frac{P_{I V}(100)}{P_{I V}(n)}}=\frac{+17.199}{+24.570}=+0.7
\end{aligned}
$$

Or:

$$
\frac{1}{p_{5}} \approx \frac{P_{V}(-100)}{P_{V}(-99)}=+1.42857143 \Rightarrow p_{5}=\frac{1}{+1.42857143}=+0.7
$$

Roots of the quintic polynomial (75) are: $p_{1}=-3.5 \cdot p_{2}=+3.0 . p_{3}=-1.8 \cdot p_{4}=+1.3 \cdot p_{5}=+0.7$ (Figure 1). 


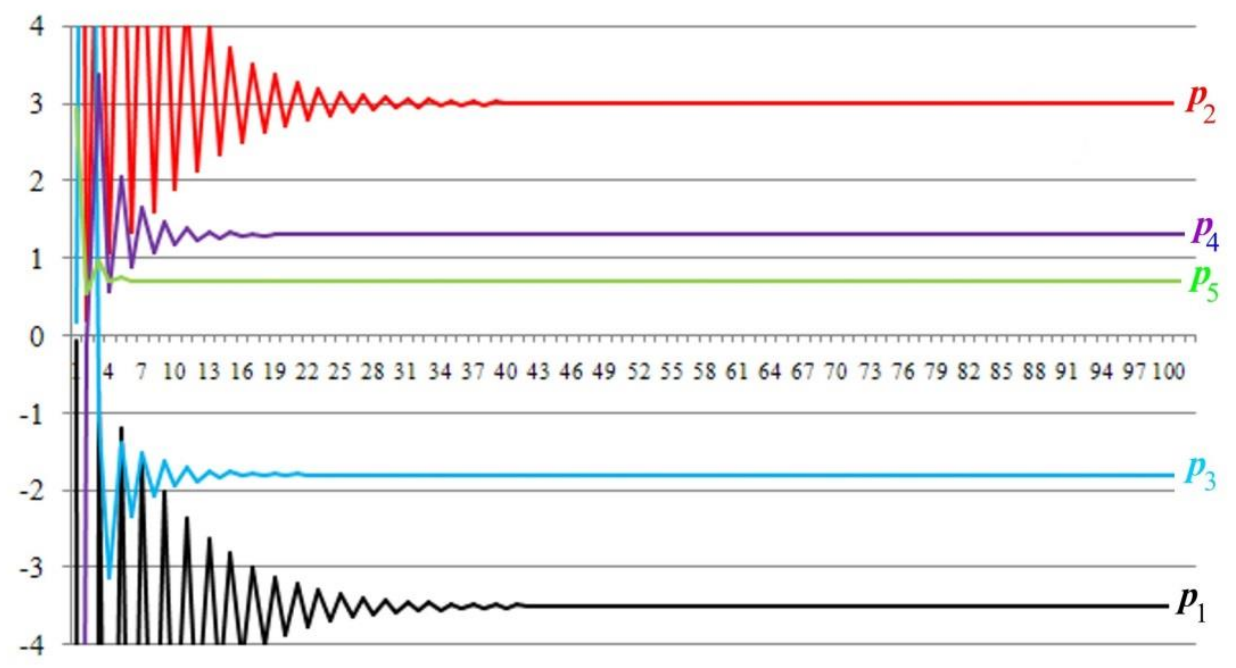

Figure 1. Convergence of the real roots.

Let us try to solve the quintic polynomial from correspondence between Professor Carl Ferdinand Degen (1766-1825) and his student Niels Abel (1802-1829). This historic polynomial from 1821 is $p(x)=x^{5}-2 x^{4}+3 x^{2}-4 x+5=0$, and it had great influence on the further focus of Niels Abel, both in approach towards Abel's impossibility theorem and elliptic functions. Pentanacci sequences of the Degen-Abel polynomial are given in Table 3:

Table 3. Pentanacci sequences of the Degan-Abel polynomial. $n \in[0,150]$.

\begin{tabular}{cccccc}
\hline$n$ & $\boldsymbol{P}_{\boldsymbol{I}}(\boldsymbol{n})$ & $\boldsymbol{P}_{\boldsymbol{I}}(\mathbf{2 n})$ & $\boldsymbol{P}_{\boldsymbol{I I}}(\boldsymbol{n})$ & $\boldsymbol{P}_{\boldsymbol{I}}(-n)$ & $\boldsymbol{P}_{I I I}(\boldsymbol{n})$ \\
\hline 0 & $5.000000 \times 10^{0}$ & $5.0000000 \times 10^{0}$ & $+10.0000000 \times 10^{0}$ & $+5.00000000 \times 10^{0}$ & $+10.0000000 \times 10^{0}$ \\
1 & $2.000000 \times 10^{0}$ & $4.0000000 \times 10^{0}$ & $+0.0000000 \times 10^{0}$ & $+0.80000000 \times 10^{0}$ & $-3.0000000 \times 10^{0}$ \\
2 & $4.000000 \times 10^{0}$ & $8.0000000 \times 10^{0}$ & $+4.0000000 \times 10^{0}$ & $-0.56000000 \times 10^{0}$ & $+11.0000000 \times 10^{0}$ \\
3 & $-1.000000 \times 10^{0}$ & $-17.0000000 \times 10^{0}$ & $+9.0000000 \times 10^{0}$ & $-0.92800000 \times 10^{0}$ & $-81.0000000 \times 10^{0}$ \\
4 & $8.000000 \times 10^{0}$ & $-88.0000000 \times 10^{0}$ & $+76.0000000 \times 10^{0}$ & $+1.19360000 \times 10^{0}$ & $+433.0000000 \times 10^{0}$ \\
5 & $-13.000000 \times 10^{0}$ & $-191.0000000 \times 10^{0}$ & $+180.0000000 \times 10^{0}$ & $+0.83168000 \times 10^{0}$ & $-663.0000000 \times 10^{0}$ \\
6 & $-17.000000 \times 10^{0}$ & $-13.0000000 \times 10^{0}$ & $+151.0000000 \times 10^{0}$ & $-0.43481600 \times 10^{0}$ & $+4723.0000000 \times 10^{0}$ \\
7 & $-82.000000 \times 10^{0}$ & $1642.0000000 \times 10^{0}$ & $+2541.0000000 \times 10^{0}$ & $-1.10606080 \times 10^{0}$ & $-29277.0000000 \times 10^{0}$ \\
\hline$\ldots$ & $\ldots$ & $\ldots$ & $\ldots$ & $\ldots$ & $\ldots$ \\
\hline 147 & $+2.8037210 \times 10^{32}$ & $-2.2895847 \times 10^{65}$ & $+4.04490510 \times 10^{64}$ & $-1.5691658 \times 10^{-7}$ & $-3.3582069 \times 10^{90}$ \\
148 & $+6.0910139 \times 10^{32}$ & $+1.4844730 \times 10^{65}$ & $+1.11278602 \times 10^{65}$ & $-1.4010369 \times 10^{-6}$ & $+1.3865365 \times 10^{91}$ \\
149 & $+1.1056579 \times 10^{32}$ & $+6.1020834 \times 10^{65}$ & $+3.06135611 \times 10^{65}$ & $-3.5544186 \times 10^{-7}$ & $-5.7247322 \times 10^{91}$ \\
150 & $+1.7314704 \times 10^{32}$ & $+1.3135825 \times 10^{66}$ & $+8.42203759 \times 10^{65}$ & $+1.0338718 \times 10^{-6}$ & $+2.3636275 \times 10^{92}$ \\
\hline
\end{tabular}

We note that:

$$
\lim _{n \rightarrow \pm \infty} \frac{P_{I}(n \pm 1)}{P_{I}(n)} \neq \text { const }
$$

e.g., the Degen-Abel polynomial is the case $\left(\rho_{1}\right)^{2}>|p|>\left(\rho_{2}\right)^{2}$. The square of the larger modulus is in the Pentanacci sequence of the second class, $\left(\rho_{1}\right)^{2}=2.75107721193586 \ldots$

$$
\frac{P_{I I}(148)}{P_{I I}(147)} \cong \frac{P_{I I}(149)}{P_{I I}(148)} \cong \frac{P_{I I}(150)}{P_{I I}(149)} \cong \ldots \cong 2.751077 \ldots
$$

The convergence of the Pentanacci third class sequence contains the product of squares of the larger modulus and the real root, $\rho_{1}^{2} p=-4.1288001348142 \ldots$

$$
\frac{P_{I I I}(148)}{P_{I I I}(147)} \cong \frac{P_{I I I}(149)}{P_{I I I}(148)} \cong \frac{P_{I I I}(150)}{P_{I I I}(149)} \cong \ldots \cong \rho_{1}^{2} p \cong-4.128800 \ldots
$$


The real root of the Degen-Abel polynomial is $p=-1.50079398604333 \ldots$

$$
\frac{\frac{P_{I I I}(148)}{P_{I I I}(147)}}{\frac{P_{I I}(148)}{P_{I I}(147)}} \cong \frac{\frac{P_{I I I}(148)}{P_{I I I}(147)}}{\frac{P_{I I}(148)}{P_{I I}(147)}} \cong \frac{\frac{P_{I I I}(150)}{P_{I I I}(149)}}{\frac{P_{I I}(150)}{P_{I I}(149)}} \cong \ldots \cong \frac{\rho_{1}^{2} p}{\rho_{1}^{2}} \cong \frac{-4.128800}{+2.751077} \cong-1.500793 \ldots
$$

The square of smaller modulus $\left(\rho_{1}\right)^{2}=1.21100557952414 \ldots$ For $P_{V}(1)=k_{0}=-5$, we obtain:

$$
\rho_{1}^{2} p \rho_{2}^{2}=P_{V}(1)=k_{0} \Rightarrow \rho_{2}^{2}=\frac{P_{V}(1)}{\rho_{1}^{2} p}=\frac{-5}{-1.507993 \ldots \cdot 2.751077 \ldots}=1.211055 \ldots
$$

We do not need the fourth-class Pentanacci values of the sequence. For $P_{I}(1)=2$ and $P_{I}(2)=4$ by (73) and (74), values $a_{1}, b_{1}, a_{2}$ and $b_{2}$ of the Degen-Abel polynomial are:

$$
\begin{aligned}
& a_{1}=+1.540781298273470 \ldots, b_{1}= \pm 0.614060422781496 \ldots \\
& a_{2}=+0.209615694748199 \ldots, b_{2}= \pm 1.080308678128320 \ldots
\end{aligned}
$$

\section{Discussion-Limitation of the New-Nacci Method}

When solving Hexanacci and Septanacci sequences, three pairs of conjugated complex roots could appear, so it can be solved using Cardano formulae. However, when solving Octanacci and Nonanacci sequences, four pairs of conjugated complex roots could appear, so it can be solved using Ferrari formulae.

When solving a Decanacci sequence, if all the roots of the decadal equation are real, the solution is straightforward. It is obvious that only in the case of the existence of five pairs of conjugated complex roots, neither Cardano formulae nor Ferrari formulae can be applied. It is clear that this problem can appear for all the sequences of the degree higher than 9 in the case of the existence of five pairs of conjugated complex roots. The famous Abel's impossibility theorem proves that limitation.

Let us consider a decadal polynomial with five pairs of conjugated complex roots $z_{1.2}, z_{3.4}, \ldots$, $z_{9.10}, z_{1.2}=a_{1} \pm i b_{1}, z_{3.4}=a_{2} \pm i b_{2}, \ldots, z_{9.10}=a_{5} \pm i b_{5}$. It is obvious that:

$$
\begin{gathered}
\lim _{n \rightarrow+\infty} \frac{D_{I}(n+1)}{D_{I}(n)} \neq \text { const, } \lim _{n \rightarrow+\infty} \frac{\frac{D_{I I I}(n+1)}{D_{I I I}(n)}}{\frac{D_{I I}(n+1)}{D_{I I}(n)}} \neq \text { const, } \lim _{n \rightarrow+\infty} \frac{\frac{D_{V}(n+1)}{D_{V}(n)}}{\frac{D_{I V}(n+1)}{D_{I V}(n)}} \neq \text { const }, \\
\lim _{n \rightarrow+\infty} \frac{\frac{D_{V I I}(n+1)}{D_{V I I}(n)}}{\frac{D_{V I}(n+1)}{D_{V I}(n)}} \neq \text { const, }, \lim _{n \rightarrow+\infty} \frac{\frac{D_{I X}(n+1)}{D_{I X}(n)}}{\frac{D_{V I I I}(n+1)}{D_{V I I I}(n)}} \neq \text { const, }
\end{gathered}
$$

Without the loss of generality, we can assume $\rho_{1}^{2}>\rho_{2}{ }^{2}>\rho_{3}{ }^{2}>\rho_{4}{ }^{2}>\rho_{5}{ }^{2}$. We get:

$$
\begin{gathered}
\lim _{n \rightarrow+\infty} \frac{\frac{D_{I I}(n+1)}{D_{I I}(n)}}{\frac{D_{I}(n+1)}{D_{I}(n)}}=\rho_{1}^{2}>\lim _{n \rightarrow+\infty} \frac{\frac{D_{I V}(n+1)}{D_{I I}(n)}}{\frac{D_{I I}(n+1)}{D_{I I I}(n)}}=\rho_{2}^{2}>\lim _{n \rightarrow+\infty} \frac{\frac{D_{V I}(n+1)}{D_{V}(n)}}{\frac{D_{V}(n+1)}{D_{V}(n)}}=\rho_{3}^{2}> \\
\lim _{n \rightarrow+\infty} \frac{\frac{D_{V I I I}(n+1)}{D_{V I I I}(n)}}{\frac{D_{V I I}(n+1)}{D_{V I I}(n)}}=\rho_{4}^{2}>\lim _{n \rightarrow+\infty} \frac{\frac{D_{X}(n+1)}{D_{X}(n)}}{\frac{D_{I X}(n+1)}{D_{I X}(n)}}=\rho_{5}^{2}
\end{gathered}
$$

Clearly:

$$
\rho_{1}^{2}=a_{1}^{2}+b_{1}^{2}, \rho_{2}^{2}=a_{2}^{2}+b_{2}^{2}, \rho_{3}^{2}=a_{3}^{2}+b_{3}^{2}, \rho_{4}^{2}=a_{4}^{2}+b_{4^{\prime}}^{2} \rho_{5}^{2}=a_{5}^{2}+b_{5}^{2}
$$


From a Decanacci sequence of the first class, it follows that:

$$
\begin{array}{ll}
D_{I}(1)=\sum_{i=1}^{5}\left(\left(a_{i}+i b_{i}\right)^{1}+\left(a_{i}-i b_{i}\right)^{1}\right), & D_{I}(2)=\sum_{i=1}^{5}\left(\left(a_{i}+i b_{i}\right)^{2}\left(a_{i}-i b_{i}\right)^{2}\right) \\
D_{I}(3)=\sum_{i=1}^{5}\left(\left(a_{i}+i b_{i}\right)^{3}+\left(a_{i}-i b_{i}\right)^{3}\right), & D_{I}(4)=\sum_{i=1}^{5}\left(\left(a_{i}+i b_{i}\right)^{4}+\left(a_{i}-i b_{i}\right)^{4}\right) \\
D_{I}(5)=\sum_{i=1}^{5}\left(\left(a_{i}+i b_{i}\right)^{5}+\left(a_{i}-i b_{i}\right)^{5}\right) &
\end{array}
$$

The system of ten equations defined by (84) and (85) cannot be solved in terms of mathematical radicals; in other words, for a polynomial with five or more pairs of conjugated complex roots, the roots cannot be determined. Please note that this result is in harmony with Abel's impossibility theorem.

However, with the known values $\rho_{1}{ }^{2} \cdot \rho_{2}{ }^{2} \cdot \rho_{3}{ }^{2} \cdot \rho_{4}{ }^{2}$ and $\rho_{5}{ }^{2}$ from (84) and (85), we obtain (87):

$$
b_{1}=\sqrt{\rho_{1}^{2}-a_{1}^{2}}, b_{2}=\sqrt{\rho_{2}^{2}-a_{2}^{2}}, b_{3}=\sqrt{\rho_{3}^{2}-a_{3}^{2}}, b_{4}=\sqrt{\rho_{4}^{2}-a_{4}^{2}}, b_{5}=\sqrt{\rho_{5}^{2}-a_{5}^{2}}
$$

Substituting the values from (87) to (86) yields new values of the quintic polynomial. New Pentanacci sequences of first class are (88):

$$
\begin{aligned}
& P_{I}^{*}(1)=D(1), P_{I}^{*}(2)=D(2), P_{I}^{*}(3)=D(3), P_{I}^{*}(4)=D(4), P_{I}^{*}(5)=D(5), \\
& P_{I}^{*}(n)=D_{I}(n)=\sum_{i=1}^{5}\left(\left(a_{i}+i \sqrt{\rho_{i}^{2}-a_{i}^{2}}\right)^{n}+\left(a_{i}-i \sqrt{\rho_{i}^{2}-a_{i}^{2}}\right)^{n}\right)
\end{aligned}
$$

With known values of Pentanacci sequences of first class, values of second (19), third (21), fourth (23) and fifth (24) class of Pentanacci sequences are available. A new quintic polynomial is (89):

$$
x^{5}+P_{I}^{*}(1) x^{4}+P_{I I}^{*}(1) x^{3}+P_{I I I}^{*}(1) x^{2}+P_{I V}^{*}(1) x+P_{V}^{*}(1)=0
$$

Real roots of the quintic polynomial (89) are values $a_{1}, a_{2}, a_{3}, a_{4}$, and $a_{5}$. From (85), we get values $b_{1}, b_{2}, b_{3}, b_{4}$, and $b_{5}$.

The second phase of the New-nacci method solves the problem of five or more pairs of conjugated complex roots. For $n$ pairs of conjugated complex roots, we get values $\rho_{i}{ }^{2}, i \in[1, n]$ from the first phase. The roots of a new $n$ th-degree polynomial (90):

$$
x^{n}+N_{I}^{*}(1) x^{n-1}+N_{I I}^{*}(1) x^{n-2}+\ldots+N_{n-1}^{*}(1) x+N_{n}^{*}(1)=0
$$

are real $a_{i}, i \in[1, n]$. In fact, with robust binomial transformations (91)

$$
\left(\left(a_{i}+i{\sqrt{\rho_{i}^{2}-a_{i}^{2}}}^{n}+\left(a_{i}-i{\sqrt{\rho_{i}^{2}-a_{i}^{2}}}^{n}\right)\right.\right.
$$

in second phase, the New-nacci method goes beyond limitations of Abel's impossibility theorem.

In the manuscript, the mathematical symbol " $>$ " is used when comparing the values of the ratio between the limits of the sequences. If we use " $\geq$ " instead of " $>$ ", it is obvious that one or more real roots have identical values, or the absolute values of some roots are identical to the square values of one or more moduli. This fact implies the slower convergence of the sequences. The problem of extremely slow convergence has been observed in the case of identical real roots of the quantic equation. Satisfactory values of the same roots (precision from $10^{-8}$ ) are obtained for Pentanacci sequences values greater than $10^{250}$. However, in this case, the roots of polynomials are apparent from monomial factorization. However, this is not considered to be a limitation of the method owing to the present performance possibilities of computers. 


\section{Conclusions}

The major advantages of New-nacci method are:

- $\quad$ The method overcomes casus irreducibilis that may appear for Cardano and Ferrari formulae.

- For calculating roots of univariate polynomials up to the 9th degree, the New-nacci method is better than the Newton-Raphson and other iterative methods [19] in the sense that there are no stationary points or poor starting points, etc.

- The New-nacci method is easier for application than Abel and Jacobi elliptic modular functions.

We can conclude that the New-nacci method:

- $\quad$ is the method for calculating roots of an arbitrary univariate polynomial based on Newton identities. Unlike the other methods that use derives or elliptic modular functions for finding the roots of univariate polynomials, the New-nacci method introduces an approach based on Newton identities for the first time;

- $\quad$ introduces a brand-new attitude for notation of Newton identities that indicates the class and degree of class for each sequence. Therefore, that approach enables the defining of Newton identities even for negative integers;

- $\quad$ is a nonlinear approximate method based on elementary mathematical operations and mathematical radicals;

- $\quad$ indices the distribution of real and complex roots before application of calculations by values of the limits of ratios of the sequences;

- $\quad$ is, in fact, a practical application of Abel's impossibility theorem and has potential limitation for determining the roots of univariate polynomials of degree higher than 9 , but the second phase of New-nacci method solves this limitation;

- $\quad$ is, in the sense of mathematical calculations, rather intensive;

- $\quad$ is extremely easy for programming and application on computers.

Although the New-nacci method is based on the work of the great masters of mathematics, e.g., Fibonacci, Kepler, Cardano, Ferrari, Newton, Abel etc., the method is declared as a new method due to the fact that it comprehends their work and is, in some way, a synthesis of their achievements and has a deep historical connotation.

Author Contributions: Methodology, I.T.; conceptualization, I.T., I.P. and Ž.S.; supervision, I.P.; visualization, I.T.; writing-original draft, I.T. and I.P.; writing—review \& editing, I.T. and Ž.S. All authors have read and agreed to the published version of the manuscript.

Funding: This research was funded by Ministry of Science and Technological Development of Serbia grant number TR 36012.

Conflicts of Interest: The authors declare no conflict of interest.

\section{References}

1. Décaillot, A.-M. Les Récréations mathématiques d'Édouard Lucas: Quelques éclairages. Hist. Math. 2014, 41, 506-517. [CrossRef]

2. Stakhov, A.; Rozin, B. The golden shofar. Chaos Solitons Fractals 2005, 26, 677-684. [CrossRef]

3. Stakhov, A.; Rozin, B. On a new class of hyperbolic functions. Chaos Solitons Fractals 2005, 23, 379-389. [CrossRef]

4. Stakhov, A. The generalized principle of the golden section and its applications in mathematics, science, and engineering. Chaos Solitons Fractals 2005, 26, 263-289. [CrossRef]

5. Kocer, E.G.; Tuglu, N.; Stakhov, A. On the m-extension of the Fibonacci and Lucas p-numbers. Chaos Solitons Fractals 2009, 40, 1890-1906. [CrossRef]

6. Tuglu, N.; Kocer, E.G.; Stakhov, A. Bivariate fibonacci like p-polynomials. Appl. Math. Comput. 2011, 217, 10239-10246. [CrossRef] 
7. Falcón, S.; Plaza, Á. On k-Fibonacci sequences and polynomials and their derivatives. Chaos Solitons Fractals 2009, 39, 1005-1019. [CrossRef]

8. Falcón, S.; Plaza, Á. The k-Fibonacci hyperbolic functions. Chaos Solitons Fractals 2008, 38, 409-420. [CrossRef]

9. Falcón, S.; Plaza, Á. On k-Fibonacci numbers of arithmetic indexes. Appl. Math. Comput. 2009, 208, 180-185. [CrossRef]

10. Tan, B.; Wen, Z.-Y. Some properties of the Tribonacci sequence. Eur. J. Comb. 2007, 28, 1703-1719. [CrossRef]

11. Bravo, J.J.; Luca, F. Coincidences in generalized Fibonacci sequences. J. Number Theory 2013, 133, $2121-2137$. [CrossRef]

12. Mansour, T.; Shattuck, M. Polynomials whose coefficients are generalized Tribonacci numbers. Appl. Math. Comput. 2013, 219, 8366-8374. [CrossRef]

13. Rybołowicz, B.; Tereszkiewicz, A. Generalized tricobsthal and generalized tribonacci polynomials. Appl. Math. Comput. 2018, 325, 297-308. [CrossRef]

14. Florek, W. A class of generalized Tribonacci sequences applied to counting problems. Appl. Math. Comput. 2018, 338, 809-821. [CrossRef]

15. Tanackov, I. Binet type formula for Tribonacci sequence with arbitrary initial numbers. Chaos Solitons Fractals 2018, 114, 63-68.

16. Da Fonseca, C.M. Unifying some Pell and Fibonacci identities. Appl. Math. Comput. 2014, 236, 41-42. [CrossRef]

17. Yang, J.; Zhang, Z. Some identities of the generalized Fibonacci and Lucas sequences. Appl. Math. Comput. 2018, 339, 451-458. [CrossRef]

18. Kılıç, E.; Arıkan, T. More on the infinite sum of reciprocal Fibonacci, Pell and higher order recurrences. Appl. Math. Comput. 2013, 219, 7783-7788. [CrossRef]

19. Luca, F.; Patel, V. On perfect powers that are sums of two Fibonacci numbers. J. Number Theory 2018, 189, 90-96. [CrossRef]

20. Chung, F.; Graham, R.; Spiro, S. Slow Fibonacci walks. J. Number Theory 2020, 210, 142-170. [CrossRef]

21. Mursaleen, M.; Nasiruzzaman, M.; Khan, F.; Khan, A. On (p,q)-analogue of divided difference and Bernstein operators. J. Nonlinear Funct. Anal. 2017, 2017, D25.

22. Srivastava, H.M.; Arjika, S.; Kelil, A.S. Some homogenous q-difference operators and the associated generalised Hahn polynomials. Appl. Set-Valued Anal. Optim. 2019, 1, 187-201.

23. Srivastava, H.M.; Ahmad, Q.Z.; Khan, N.; Kiran, S.; Khan, B. Some applications of higher-order derivatives involving certain subclass of analytic and multivalent functions. J. Nonlinear Var. Anal. 2018, 2, 343-353.

24. Connon, E. Abel's proof: An essay on the sources and meaning of mathematical unsolvability. Hist. Math. 2006, 33, 371-372. [CrossRef]

(C) 2020 by the authors. Licensee MDPI, Basel, Switzerland. This article is an open access article distributed under the terms and conditions of the Creative Commons Attribution (CC BY) license (http://creativecommons.org/licenses/by/4.0/). 